\title{
Rare case of intra-testicular adenomatoid tumour
}

\author{
Filippo Migliorini ${ }^{1}$, Roberto Baldassarre ${ }^{1}$, Walter Artibani ${ }^{1}$, Guido Martignoni ${ }^{2}$, Matteo Brunelli ${ }^{2}$ \\ ${ }^{1}$ Urology Department, University Hospital, Ospedale Policlinico, Azienda Ospedaliera Integrata, Verona, Italy; \\ ${ }^{2}$ Department of Pathology and Diagnostic, University Hospital, Ospedale Policlinico, Azienda Ospedaliera Integrata, Verona, Italy.
}

\begin{abstract}
Summary Adenomatoid tumors are rare benign neoplasms considered of mesothelial origin. They are usually asymptomatic and slow growing masses. They account for 30\% of paratesticular tumors and very rarely involve the testicular parenchyma. Only ten such cases have been reported in the literature so far. Ideal treatment should be excision of the tumor avoiding orchidectomy. Nevertheless, because of the rarity of the lesion and the difficulty of distinguishing it from malignancy, radical orchidectomy is often performed. We describe a case of a 31 years old caucasian man who presented with a moderately symptomatic left testicular mass, normal tumor markers and normal sex hormones levels. The ultrasound showed an hypoechoic intratesticular nodule of $0.8 \mathrm{~cm}$ in diameter. The patient underwent intraoperative frozen section of the nodule which could not exclude malignancy with certainty. A radical orchiectomy was therefore performed. Subsequent definitive histological and molecular report described an adenomatoid tumor involving the parenchyma of the testis.
\end{abstract}

KEY WORDS: Adenomatoid tumour; Testis; Pathologic findings; Orchifuniculectomy.

Submitted 7 February 2014; Accepted 28 February 2014

\section{INTRODUCTION}

Adenomatoid tumors are relatively uncommon benign tumors of mesothelial origin, usually occurring in the genital tract of both males and females.

Extragenital localization is rare and has been reported in adrenal glands, heart, mesentery, lymph nodes and pleura. Adenomatoid tumors are responsible for 30\% of all paratesticular masses and are most commonly found at the head of the epididymis. Exceptionally, these tumors involve the testicular parenchyma and only ten cases have been previously reported in the literature (1-2). The ideal treatment should be excision of the nodule with preservation of the testicle. This is not always possible because the morphological features on the frozen section don't allow a certain exclusion of malignancy.

Case Report and Figures are posted in Suppementary materials on www.aiua.it.

\section{Discussion}

Here we report a case of adenomatoid tumor of the testis with intratesticular growth and describe the ultrasound, gross and pathologic characteristics of this entity. To the best of our knowledge, only 10 cases have been previously reported in the literature (3).

Adenomatoid tumors are rare benign neoplasms occurring in both sexes and very rarely present as intratesticular masses. These tumors originate from the tunica albuginea but might also be found in the tunica vaginalis and rete testis. They present as well-circumscribed unencapsulated tumors with tan white cut surface and might be indistinguishable from seminoma (1-2).

Microscopically, they show different morphological patterns as tumor cells can form solid cords, nests, glandular-like spaces or tubules. A typical feature of the neoplastic cells is the presence of vacuolated cytoplasm and cytologic atypia with absence of mitosis. The stroma is usually fibrous although a smooth muscle component might be present. Even though tumors may focally infiltrate between testicular tubules, such finding should not be considered as evidence of malignancy. A useful hint for the diagnosis of these lesions is the presence of lymphoid aggregates often localized at the periphery of the tumor. Interestingly, this characteristic is usually lacking in tumors arising in females.

The immunophenotipic profile of adenomatoid tumors shows positivity for pancytokeratins, podoplanin, WT1 and calretinin and can be very useful in the differential diagnosis with neoplasms that may resemble adenomatoid tumors, namely Yolk sac tumor (negative for WT1 and calretinin), Leydig cell tumor (negative for WT1) and metastatic carcinoma (1-2). Also, negativity for vascular markers like CD34 helps in excluding tumors of vascular origin. A difficult differential diagnosis is represented by malignant mesothelioma which is distin-

No conflict of interest declared 
guished from adenomatoid tumor for its larger size, invasive growth pattern and involvement of adjacent structures. Since adenomatoid tumors have never shown malignant behavior, the aim in treating these masses is to prevent unnecessary orchidectomy thus preserving fertility and testosterone production. In this regard, accurate imaging and preoperative assessing of serum tumor markers like alpha-fetoprotein, LDH and beta-HCG might help in excluding a malignant lesion.

Intraoperative frozen section can also help in determining benignity of a testicular mass; accordingly, the value of intraoperative biopsy evaluation has become more popular in the last years, allowing organ-sparing procedure for non-malignant lesions. However, in the literature, only in one case of adenomatoid tumor with intratesticular growth was performed a conservative tumorectomy. This proves the extreme difficulty for a pathologist to rule out malignancy on frozen section in the case of an adenomatoid tumor with intratesticular growth. In our case, a diagnosis of benignity on the intraoperative biopsy was not possible and an orchidectomy was carried out. In conclusion, adenomatoid tumor with intratestic- ular growth is a rare neoplasm that can show different morphological features and therefore represents a diagnostic challenge, especially on frozen section.

The pathologist must be aware of this entity when evaluating intraoperative biopsies of testicular masses. It must be stated, however, that ruling out malignancy is often not possible and orchidectomy cannot be avoided most of the times

\section{REFERENCES}

1. Borislav AA, Lauren FX, Jonathon EH, et al. Adenomatoid Tumor of the Testis With Intratesticular Growth: A Case Report and Review of the Literature. Int J Surg Pathol. 2011; 19:838-842.

2. Pacheco AJ, Torres JL, De La Guardia FV, et al. Intraparenchymatous adenomatoid tumor dependent on the rete testis: A case report and review of literature. Indian J Urology. 2009; 25:126-128

3. Alexiev BA, Xu LF, Heath JE, et al. Adenomatoid tumor of the testis with intratesticular growth: a case report and review of the literature. Int J Surg Pathol. 2011; 19:838-842.

Figure 1.

Intraoperative biopsy. Note the nodule with an intra-testis localization (left side). Normal testicular parenchyma inked on the right side (H\&E, 4X).

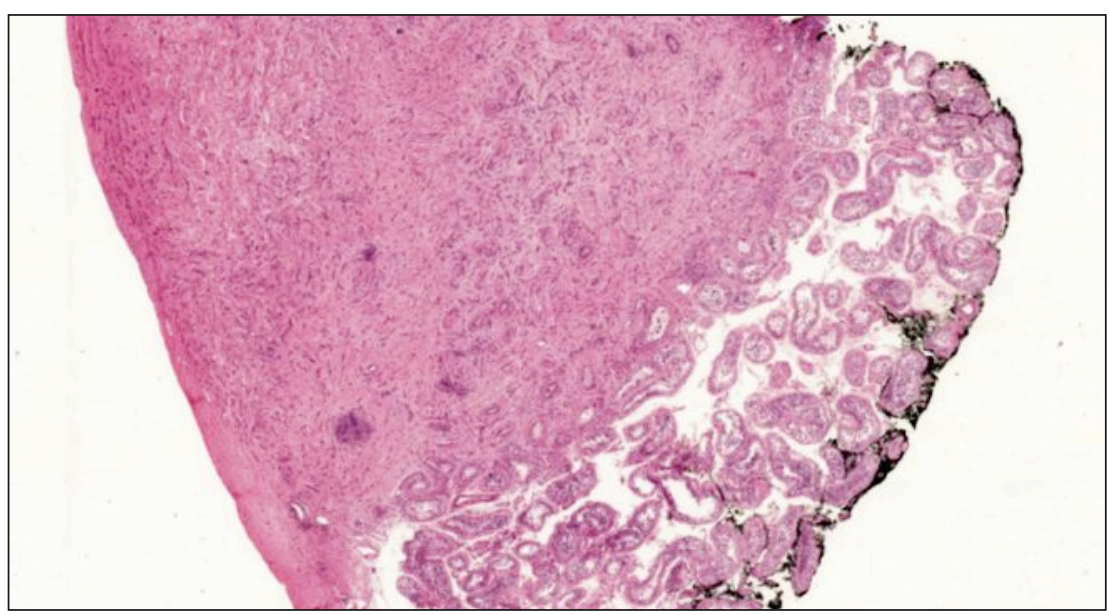

\section{Correspondence}

Filippo Migliorini, MD (Corresponding Author)

filippo.migliorini@ospedaleuniverona.it

Roberto Baldassarre, MD

roberto.baldassarre@ospedaleuniverona.it

Walter Artibani, MD

walter.artibani@univr.it

Urology Department, University Hospital,

Ospedale Policlinico, Azienda Ospedaliera Integrata,

P.le Ludovico Scuro 10 - 37134 Verona, Italy

Guido Martignoni, MD

guido.martignoni@univr.it

Matteo Brunelli, MD

matteo.brunelli@univr.it

Department of Pathology and Diagnostic, University Hospital,

Ospedale Policlinico, Azienda Ospedaliera Integrata,

P.le Ludovico Scuro 10 - 37134 Verona, Italy 